\title{
La responsabilidad social corporativa y el desempeño financiero: un análisis en empresas mexicanas que cotizan en la bolsa
}

María del Mar
Alonso-Almeida
Universidad Autónoma de
Madrid
mar.alonso@uam.es
Marthadel Pilar
Rodríguez,García
Universidad Autónoma de
Nuevo León
marthadelpilar2000@yahoo.com

Klender Aimer

Cortez Alejandro

Universidad Autónoma de Nuevo León

kcortez@facpya.uanl.mx

José Luis Abreu Quintero Universidad Autónoma de Nuevo León spentamex@hotmail.com

\section{Resumen}

La responsabilidad social empresarial (RSE) está cobrando gran relevancia en las últimas dos décadas como una estrategia que puede contribuir al desempeño financiero de la empresa y a construir un futuro general más equitativo y sostenible; sin embargo, en lo que tiene que ver con el desempeño financiero, hasta ahora los resultados obtenidos por la doctrina han sido mixtos. Este trabajo analiza el impacto en el desempeño financiero de las empresas mexicanas cotizadas que han obtenido el distintivo de empresas socialmente responsables. Los resultados obtenidos muestran que existe una relación positiva entre el desempeño financiero y la obtención de dicho distintivo en las variables financieras ROE y ROA, en las utilidades por acción (UPA) y en el ratio de precio entre valor de libros (P/VL). La evidencia encontrada proporciona algunas recomendaciones para las empresas mexicanas y los legisladores en este ámbito.

Palabras clave: RSE, desempeño financiero, estrategia, distintivo ESR. 


\title{
Corporate social responsibility and financial performance: an application to Mexican listed companies
}

\begin{abstract}
Corporate Social responsibility (CSR) is becoming very important in the last two decades as a strategy that can contribute to the company and to build a more equitable and sustainable overall future financial performance. However, in what has to do with the financial performance, so far, the results obtained by the doctrine have been mixed. This paper analyzes the impact on the financial performance of Mexican listed companies that have earned the distinction of socially responsible companies. The results obtained show that there is a positive relationship between financial performance and obtaining such distinction as shown in financial variables like ROE and ROA, earnings per share (UPA) and the price ratio between books (P/VL) value. Evidence found provides some recommendations for Mexican companies and policy-makers in this field.
\end{abstract}

Keywords: CSR, financial performance, strategy, distinctive of CSR.

\section{Introducción}

Tras los primeros diez años del siglo XXI parece ampliamente aceptado que la creación de valor se debe producir no sólo para el accionista, sino también para el resto de los partícipes y agentes sociales, según la función que cumpla cada uno (Bueno-Campos, 2004).

Este planteamiento se conoce en la literatura como el enfoque de los stakeholders, superado el enfoque financiero clásico de los shareholders. Milton Friedman (1962) encarna el enfoque shareholder que se resume en dos principios: 1) el único público al que la empresa debe rendir cuentas es a sus accionistas; 2) el único objetivo que debe buscar la empresa es la maximización del beneficio o valor de la empresa. Por el contrario, el enfoque stakeholder engloba a la totalidad de grupos que tienen interés en la empresa que ésta tiene que equilibrar en su propio beneficio (Freeman, 1994). Estos grupos estarían constituidos por los accionistas, los empleados, los proveedores tanto de capital financiero como de bienes y servicios, el estado y la sociedad en su conjunto (Alonso-Almeida, 2007). Por tanto, se puede decir que la empresa es un nexo de contratos implícitos basados en la confianza y la reputación que intenta equilibrar todos los intereses involucrados en ella, tanto externos como internos (Alonso-Almeida, 2009; Kramer y Tyler, 1996; Lane y Bachmann, 2000). 
Una vez reconocida la existencia de unos stakeholders que pueden contribuir al éxito de la organización (Clarkson, 1995; Post et al., 2002), las empresas durante los últimos treinta años han comenzado a hacer frente a sus demandas, unas veces debido a la fuerza de la legislación y otras de forma voluntaria o por la presión explícita de ciertos grupos. A todas las acciones dirigidas a satisfacer las necesidades de los diferentes stakeholders se denomina responsabilidad social empresarial, en adelante RSE (Clarkson, 1995; Wood, 1991).

En la actualidad existe una gran discusión tanto en el mundo empresarial como en el académico sobre el aporte "real" de la RSE a la empresa y a su desempeño financiero. Hay autores que afirman que el desarrollo de la RSE por parte de las empresas es uno de los principales inductores de la mejora del desempeño financiero (Annandale y Taplin, 2003; Bull, 2003; Kiernan, 2001; Prakash, 2000; Sharma, 2000; Tullberg, 2005). Mientras que en algunos estudios se ha encontrado una relación positiva entre los impactos de la RSE en la empresa y el desempeño financiero (Derwall et al., 2005; Graves y Waddock, 1994; Griffin y Mahon, 1997; Lee y Faff, 2009; Margolis y Walsh 2001; Orlitzky et al., 2003; Petersen y Vredenburg, 2009), en otros esta relación es difusa (Bragdon y Marlin, 1972; Brammer et al., 2006; Friedman, 1970). Por eso, se hace necesario investigar más en este campo. Especialmente, la escasez de estudios es más evidente en los países en vías de desarrollo.

Por tanto, este artículo tiene dos objetivos principales. En primer lugar, contribuir a extender el conocimiento del impacto de la RSE en las empresas desde el punto de vista financiero sin prescindir del resto de los involucrados en las empresas, los stakeholders. En segundo lugar profundizar en el desarrollo de la RSE en México, ya que apenas existe literatura aplicada a este país. Adicionalmente, se propondrán algunas recomendaciones para la práctica empresarial y los legisladores.

El artículo se divide en seis secciones, incluyendo la introducción; se hace una revisión de los principales indicadores de RSE en el mundo; más adelante se describe la responsabilidad social corporativa en México; en la siguiente sección se presentan antecedentes de las relaciones encontradas entre RSE y desempeño financiero; posteriormente, se explican las variables y el modelo; después se dan los resultados; finalmente, en las últimas dos secciones, se presentan las conclusiones y la bibliografía. 


\section{Indicadores de la responsabilidad social corporativa (RSE)}

Haigh y Jones (2006) identificaron seis grupos de factores que aparecían en la literatura como promotores de la responsabilidad social: 1) factores interorganizacionales, 2) dinámica competitiva, 3) inversores institucionales, 4) poder de los consumidores finales, 5) reguladores gubernamentales y 6) organizaciones no gubernamentales. La presión de estos factores ha sido la responsable del desarrollo de la RSE que se ha materializado en los diferentes indicadores y acciones que se van a analizar a continuación.

Algunas investigaciones han puesto de manifiesto que las primeras aproximaciones a la RSE se produjeron en términos de políticas y procedimientos de gestión de recursos humanos (Winstanley et al., 1996). Así, se puede considerar que las primeras inversiones sociales e indicadores que se utilizaron en el interior de las empresas se realizaron en los empleados de las propias empresas, mediante el desarrollo de planes de formación, planes de desarrollo profesional, protección en el trabajo y mejoras en su calidad de vida. Bartels et al. (1998) realizaron un estudio en el que relacionaban el clima ético de la empresa y las prácticas de recursos humanos; estos autores encontraron una relación positiva en un incremento de la ética en la empresa mediante la formación de los empleados, la adopción de códigos éticos y de buenas prácticas y la aplicación de los estándares de salud y seguridad en el trabajo.

Waddock (2004) en un análisis sobre la evolución del concepto de RSE, desde los años setenta hasta la actualidad y desde el interior de la empresa hacia afuera, explicó que las prácticas que habían sido adoptadas inicialmente desde los años sesenta hasta finales de esta década estaban basadas en su relación con otras empresas y con la comunidad mediante programas de filantropía y acciones de voluntariado. A mediados de la década de los ochenta, las estructuras de las empresas se transforman para hacer una política de RSE más cercanas a los stakeholders: departamentos de atención a los clientes, relación con los inversores, introduciendo relaciones públicas, entre otros. A partir de finales de los años ochenta las empresas vieron la necesidad de incorporar códigos de conducta ética a sus actividades. Igualmente, era importante informar públicamente sobre la labor social de la compañía, ya que esta información servía para poner una buena base comunicativa entre la empresa y sus stakeholders (Dierkes y Antal, 1985). En los años noventa, las quejas de los ecologistas y la importancia creciente sobre los problemas medioambientales hicieron que se comenzaran a tomar medidas de este tipo (acción medioambiental) 
y divulgar los informes medioambientales y, posteriormente, los informes de acción social de forma individualizada. En la actualidad, el reporte medioambiental y el reporte social, de forma individualizada, se suelen ver como herramientas de diálogo entre la empresa y sus stakeholders, lo que puede hacer que la empresa se sienta presionada a efectuar cambios debido a que expone públicamente sus actos y, por tanto, puede ser examinada y observada en cuanto a lo que no "hace bien" (Greenwood, 2007).

Adicionalmente, existen estándares internacionales que recogen todas estas iniciativas sobre RSE — GRI, ISO26000, AA1000- y las empresas pueden voluntariamente adherirse a ellas. El Global Reporting Iniciative (GRI) es una iniciativa global para la difusión de la información económica, medioambiental y social de las empresas, la cual se efectúa mediante la creación de unas guías estándar en el nivel internacional (GRI, 2009). La norma de aseguramiento AA1000 es una norma que ayuda a las empresas a introducir procesos en su interior que involucren a todas las partes interesadas en la empresa e indicadores asociados (Accountability, 2003). La norma ISO26000 es una norma voluntaria y no certificable que proporciona "una guía práctica tendiente a hacer operativa la responsabilidad social, identificar y comprometer a los stakeholders, y reforzar la credibilidad de los informes y reclamos realizados sobre Responsabilidad Social" (ISO, 2006). Además de las medidas internas, otros organismos externos han medido las acciones que en el ámbito de la RSE han realizado las empresas. Igalens y Gond (2005) recogieron los principales indicadores de RSE externos utilizados por los estudios empíricos y los clasificaron en índices de polución, encuestas, indicadores de reputación corporativa y datos producidos por organizaciones de medición externas a la empresa. Las principales características de estos indicadores se recogen en el cuadro 1. 


\section{Cuadro 1}

\section{Principales indicadores externos de RSE recogidos por la literatura}

\begin{tabular}{|c|c|c|c|c|}
\hline $\begin{array}{l}\text { Tipo de } \\
\text { medida }\end{array}$ & Quién lo realiza & Objetivo & Ejemplo & $\begin{array}{l}\text { Principal } \\
\text { característica/ } \\
\text { Problema }\end{array}$ \\
\hline $\begin{array}{l}\text { Índices de } \\
\text { polución }\end{array}$ & $\begin{array}{l}\text { Agente externo a } \\
\text { la empresa }\end{array}$ & $\begin{array}{l}\text { Asegurar la } \\
\text { objetividad }\end{array}$ & $\begin{array}{l}\text { Toxic Release } \\
\text { Inventory (TRI) }\end{array}$ & $\begin{array}{l}\text { Aplica a } \\
\text { determinado tipo de } \\
\text { industrias }\end{array}$ \\
\hline $\begin{array}{l}\text { Encuestas } \\
\text { específicas }\end{array}$ & $\begin{array}{l}\text { Estudios } \\
\text { académicos }\end{array}$ & $\begin{array}{l}\text { Medir y materializar } \\
\text { los diferentes } \\
\text { constructos de la } \\
\text { RSE. } \\
\text { Medir la actitud de } \\
\text { la empresa hacia la } \\
\text { RSE. }\end{array}$ & $\begin{array}{l}\text { Estudios de } \\
\text { diversos autores: } \\
\text { Aupperle } \text { et al., } \\
\text { (1985); Maignan } \\
\text { et al., (1999); } \\
\text { Maignan y Ferrer, } \\
\text { (2001) }\end{array}$ & $\begin{array}{l}\text { Un investigador } \\
\text { recoge la } \\
\text { información de la } \\
\text { compañía }\end{array}$ \\
\hline $\begin{array}{l}\text { Índices de } \\
\text { reputación } \\
\text { corporativa }\end{array}$ & $\begin{array}{l}\text { Entidad externa a } \\
\text { la empresa }\end{array}$ & $\begin{array}{l}\text { Medir la opinión que } \\
\text { terceros externos a } \\
\text { la empresa tienen } \\
\text { sobre ella }\end{array}$ & Fortune & $\begin{array}{l}\text { El principal } \\
\text { problema es la } \\
\text { subjetividad de los } \\
\text { entrevistados }\end{array}$ \\
\hline $\begin{array}{l}\text { Datos } \\
\text { producidos } \\
\text { por entidades } \\
\text { de medición } \\
\text { externas }\end{array}$ & $\begin{array}{l}\text { Datos producidos } \\
\text { por entidades } \\
\text { especializadas en } \\
\text { RSE }\end{array}$ & $\begin{array}{l}\text { Recoger y medir la } \\
\text { información que la } \\
\text { empresa proporciona } \\
\text { sobre RSE }\end{array}$ & $\begin{array}{l}\text { KLD en Estados } \\
\text { Unidos }\end{array}$ & $\begin{array}{l}\text { Depende del modo } \\
\text { de medición de cada } \\
\text { una de las entidades }\end{array}$ \\
\hline
\end{tabular}

Fuente: Elaboración propia a partir de Igalens y Gond (2005).

Finalmente, el concepto de RSE en la actualidad ha adquirido una dimensión más amplia y representa el compromiso adquirido de forma voluntaria por la empresa, más allá de sus obligaciones legales, y que se orienta a la satisfacción de sus stakeholders mejorando la situación laboral de sus empleados, adecuando los precios de los productos y servicios de calidad y supervisando el comportamiento ético con todos los agentes sociales; además, es importante considerar el medio ambiente y el desarrollo de la comunidad (Méndez, 2005; Porto y Castromán, 2006).

\section{La responsabilidad social corporativa (RSE) en México}

La escasa literatura existente en México sobre RSE se ha alineado en dos direcciones. Por un lado, una parte de la doctrina se ha focalizado en describir el concepto de RSE y realizar análisis comparativos con otros países; en este sentido Porto y Castromán (2006) hacen un análisis comparativo entre España y México, en donde 
encuentran que para el 2005 existía una mayor aceptación del concepto de RSE en México que en España, mientras en nuestro país se habían otorgado 25 distintivos de RSE en las empresas, en España únicamente eran 13 los distintivos otorgados.

Por otro lado, otra parte de la doctrina se ha centrado en estudiar el desarrollo de la RSE en México analizando sus peculiaridades propias. Entre este tipo de estudios destaca el de Barroso (2008) que muestra que los directores de 40 empresas de Mérida, Yucatán, utilizan el término de RSE para mejorar la imagen olvidando la conservación del medioambiente, por lo que no se aplica el concepto de RSE en sentido estricto. Muller y Kolk (2009) muestran que las actividades de RSE en compañías de autopartes mexicanas son similares a las actividades sociales en los países en desarrollo.

En México, existen instituciones como AliaRSE, constituida en 2007 y comprometida e interesada en promover la responsabilidad social empresarial en México, que integra la alianza de la Confederación Patronal de la República Mexicana, del Consejo Coordinador Empresarial de la Confederación de Cámaras Industriales, de la Confederación Unión Social de Empresarios Mexicanos, del Centro Mexicano para la Filantropía y de Impulsa. Expresa que uno de los logros más importantes de la alianza es haber logrado el consenso de un concepto y marco ideológico común en México; en este sentido, han llegado a la conclusión de que la RSE puede ser entendida "como el compromiso consciente y congruente que asume el empresario y la empresa de cumplir integralmente con la finalidad de la empresa tanto en lo interno, como en lo externo, considerando las expectativas de todos sus participantes en lo económico, social o humano y ambiental, demostrando el respeto por los valores éticos, las personas, las comunidades y la construcción del bien común con justicia social”. Por otra parte, Cemefi —un organismo localizado en la Ciudad de México cuyo propósito es el de apoyar a la empresa a iniciar en el camino de la RSE, a mejorar o medir sus prácticas - ha expuesto que se identifica con el concepto expresado por AliaRSE; distingue anualmente a las empresas que cumplen lineamientos en cuatro temáticas igualmente importantes (ética empresarial, vinculación de la empresa con la comunidad, calidad de vida en la empresa y cuidado y conservación del medio ambiente) para hacerse acreedoras al distintivo de Empresas Socialmente Responsables (ESR), el cual se otorga anualmente. Cabe destacar que las empresas que cotizan en la Bolsa Mexicana de Valores (BMV), una vez que logran el distintivo, lo siguen conservando. 


\section{RSE y desempeño financiero}

Cada vez más el tema de RSE tiene que ser alineado o relacionado con el desempeño de la empresa, pues en la medida que el administrador haga una alineación con el desempeño financiero, los accionistas confiarán en este tópico y de esta forma se tendrá impacto social. Los estudios realizados hasta ahora, que han intentado relacionar las prácticas de RSE y el desempeño financiero de la empresa, han encontrado resultados mixtos.

Según Peloza (2009), los estudios relacionados con el tema de RSE desde hace tres décadas muestran que existe una relación positiva entre éste y desempeño financiero, aunque no ha sido demostrada empíricamente porque las investigaciones que se han realizado mayoritariamente son de estudios de caso.

Margolis et al. (2007) y Orlitzky (2007) encuentran que los profesionales de la RSE encuentran resultados más positivos en la relación de RSE y desempeño financiero. En particular, Peloza (2009) realiza un análisis sobre los resultados que se han tenido desde la década de los setenta y divide los hallazgos en dos: 1) estudios académicos y 2) estudios de profesionales. Encuentra que los investigadores consideran que el $59 \%$ de los estudios reportados muestran relaciones positivas entre el desempeño financiero y RSE; 27\%, resultados mixtos; y 14\%, relaciones negativas; en tanto que los profesionales encuentran que el $77 \%$ de los reportes muestran resultados positivos entre RSE y actuación financiera; $10 \%$, reportes mixtos o neutros; y 13\%, conclusiones negativas.

Otros estudios que han encontrado una relación positiva entre RSE y desempeño financiero de la empresa son los de Graves y Waddock (1994), Griffin y Mahon (1997), Margolis y Walsh (2001), Orlitzky et al. (2003), Derwall et al. (2005), Petersen y Vredenburg (2009), Lee y Faff (2009), entre otros.

Graves y Waddock (1994) identifican que los inversionistas institucionales prefieren alinear RSE a organizaciones socialmente responsables que a las que no lo son. Prueba de ello es que actualmente los inversionistas se encuentran comprometidos con el tema de RSE; por ejemplo, en invertir en el índice de sustentabilidad del Dow Jones y algunas otras invierten en este índice para mejorar su reputación.

Griffin y Mahon (1997) estudiaron el desempeño social y financiero de seis empresas de la industria petroquímica de 1990 a 1992; encontraron que existe relación 
positiva entre el índice KLD y el índice Fortune con métricas contables. Margolis y Walsh (2001) encontraron ciento veintidós estudios publicados entre 1971 y 2001; examinaron la relación positiva entre responsabilidad social empresarial y el desempeño financiero. Por otra parte, Orlitzky et al. (2003) demuestran que las medidas contables tienen una correlación mayor con el desempeño social que las medidas financieras. En Derwall et al. (2005) se argumenta que las inversiones en RSE dirigen a la empresa a un desempeño financiero a largo plazo mayor debido a la mejora en la reputación y en la calidad de sus administradores y empleados. Petersen y Vredenburg (2009) realizan un estudio en el sector de petróleo mediante la metodología de caso en Canadá y señalan que existe evidencia que demuestra que el comportamiento de una empresa socialmente responsable empieza agregando valor económico y que los esfuerzos de invertir en proyectos en temas de RSE se reconoce y premia en los mercados de capitales, con una mayor rentabilidad económica. Por último, Lee y Faff (2009) encuentran que, en Europa y en Estados Unidos, los inversionistas apuestan por empresas que invierten en RSE.

Sin embargo, aparecen estudios que señalan que existe una relación inversa entre la RSE y el desempeño de la empresa (Bradgon y Marlin, 1972; Brammer et al., 2006; Friedman, 1970), especialmente se atribuyen estos resultados a los altos costos en que se incurren para mejorar el desempeño ambiental.

Ullman (1985) menciona que, debido a la gran cantidad de variables que intervienen en la RSE y el desempeño financiero, no hay razón para suponer una relación directa. Lee y Faff (2009) encuentran que las métricas basadas en la contabilidad y las basadas en el mercado no tienen relación con las medidas de la RSE. En el caso de países emergentes, Lima et al., (2010) encuentran que para el caso de Brasil existe una relación inversa entre la RSE y el desempeño financero; el estudio cuenta con una muestra de 78 firmas brasileñas analizadas durante el periodo que va de 2001 al 2006 y argumentan que esta relación negativa se puede deber a que los inversionistas no se sienten motivados a invertir en empresas responsables por aspectos culturales. Estos mismos autores mencionan que el tema de RSE debe considerarse desde un punto de vista publicitario y con efectos a mediano y largo plazo en el rendimiento, ya que a corto plazo los administradores, con base en los resultados de Brasil, se verían desalentados a destinar dinero en inversiones responsables.

Por otra parte, existen estudios que encuentran relaciones neutras; por citar un caso, encontramos el trabajo de Aras et al. (2010) que encuentran nula relación 
en el caso de Estambul entre RSE y desempeño financiero de cien empresas que cotizan en el mercado accionario entre 2005 y 2007.

\section{Metodología de análisis}

\section{Muestra}

Para determinar la situación actual de las empresas que realizan prácticas de RSE usaremos la base de datos de Cemefi, que otorga anualmente el distintivo de Empresas Socialmente Responsables (ESR). El periodo de estudio son los años comprendidos de 2001 al 2009 y la muestra está formada por empresas que han mantenido y renovado anualmente el distintivo de ESR y, además, que cotizan en la Bolsa Mexicana de Valores (BMV). En total son 20 empresas que representan el $15 \%$ de las empresas que cotizan en la BMV y sólo el 5\% de las empresas que son responsables socialmente de acuerdo con el distintivo de ESR.

En la figura 1, se observa el número de distintivos de ESR que se han entregado durante el periodo de estudio. Como se puede ver, el máximo crecimiento se observa en el 2002 con $65 \%$ de tasa de crecimiento, mientras que la más baja de todo el periodo se observa en el 2009 con $25 \%$ de crecimiento. Cabe destacar que, en promedio, durante este periodo se han otorgado 128 distintivos anuales con una desviación estándar de 116 distintivos.

\section{Figura 1}

\section{Empresas con distintivo de ERS. Número de empresas con distintivo de ESR}

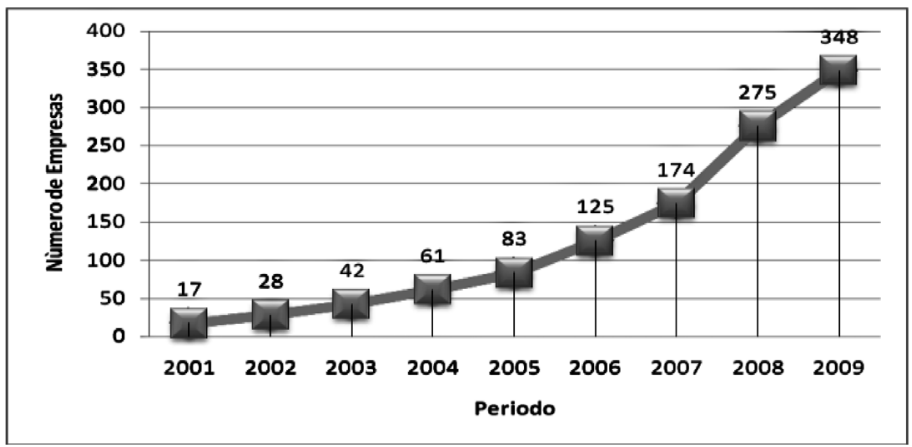

Fuente: Confederación Unión Social de Empresarios Mexicanos del Centro Mexicano de Filantropía (2011). 


\section{Variable de RSE}

Para esta variable se utilizó una dummie en donde 1 refleja que la empresa tiene el distintivo de RSE otorgado en un año por Cemefi y 0 que no lo tiene.

\section{Variables de desempeño financiero}

En este trabajo dividimos las variables de desempeño con el fin de facilitar el estudio en tres partes. La primera se basa en variables obtenidas a partir de información contable, las que se utilizan en este estudio son rendimiento sobre activos (ROA), rendimiento sobre capital (ROE), las utilidades por acción (UPA) y el crecimiento de las utilidades, que son las variables más utilizadas por la doctrina en este tipo de estudios para medir el desempeño financiero (Peters y Mullen, 2009; Waddock y Graves, 1997).

Por otra parte, existen métricas que se calculan con datos de los estados financieros y con información del mercado. Entre una gran variedad de métricas encontramos el precio entre valor en libros (P/VL) (Lima et al., 2010) y el precio entre utilidades por acción (P/UPA). Rappaport (1998) señala que estas medidas son mejores que las contables por considerar elementos del mercado; sin embargo, están basadas en la contabilidad histórica y, por tanto, comparten sus defectos.

Por último, se encuentran las métricas que se calculan con base en el precio de la acción, usadas en Schnietz y Epstein (2005), y buscan responder si se crea o no riqueza de un periodo a otro; en este caso se utiliza el rendimiento en precios.

\section{Variables de control}

Utilizaremos variables de control para moderar los efectos del modelo (Waddock y Graves, 1997). En este estudio, se utiliza el activo como indicador del tamaño de la empresa (Peters y Mullen, 2009; Lima et al, 2010). Según Lima et al. (2010), el tamaño de la empresa medido de esta forma es una importante variable de control, pues puede tener influencia en las acciones de RSE. Las empresas pequeñas pueden tener menos capacidad para sostener comportamientos de carácter social en comparación con las grandes. Otra medida de control propuesta en el estudio de Lima et al. (2010) es el riesgo medido a través de la capacidad de pago de la empresa mediante la proporción el pasivo (P) al activo (A) de la empresa. 


\section{Modelo}

Debido a la heterogeneidad de nuestras observaciones en relación con el periodo de estudio y con el número de empresas, seleccionamos una metodología de datos panel para series de tiempo de corte transversal, ya que contamos con información de 19 empresas de la Bolsa Mexicana de Valores (BMV) para el periodo de 2001 al 2009. El modelo es parecido al estimado en Lima et al. (2010) y se especifica a través de la siguiente ecuación de regresión:

$$
D F_{i t}=\alpha_{0}+B_{1} R S E_{i t}+B_{2} T_{i t}+B_{3} R_{i t}+e_{i t}
$$

donde, $\mathrm{i}=1,2, \ldots, 19$ unidades de corte transversal (empresas de la BMV seleccionadas) y $\mathrm{t}=1,2, \ldots, 9$ años (2001-2009).

Las variables del modelo son:

$D F_{i t}=$ Esta variable puede estar representada por ROA, ROE, UPA, crecimiento de utilidades, precio entre valor en libros, precio entre UPA y rendimiento de mercado.

$R S E_{i t}=$ Variable dummie que toma el valor de 0 para empresas que no tienen distintivo en un año de RSE y 1 en empresas que tienen el distintivo en un año en particular.

$T_{i t}=$ Variable de control que supone el tamaño de la empresa medido como el logaritmo de los activos.

$R_{i t}=$ Mide el riesgo de la empresa a través del endeudamiento de la empresa (P/A).

Los parámetros $\alpha$ it y $\beta$ i son la constante y la pendiente de la línea de regresión; el término $e_{i t}$ es el error de estimación con $e_{i t} \mathrm{~N}(0,1)$. Para estimar los coeficientes betas $(\beta \mathrm{i})$, se optó por el método de mínimos cuadrados generalizados (MCG), bajo la corrección de corte transversal ponderado, en lugar de mínimos cuadrados ordinarios (MCO) en virtud de que este último asigna igual peso a cada observación sin importar la variabilidad de los datos entre las distintas unidades o empresas (como se explicará en el cuadro 2), mientras que el primero tiene en cuenta esa información explícitamente y, por consiguiente, es capaz de producir un mejor estimador lineal insesgado. Además, se seleccionó MCG porque, frecuentemente 
en los modelos de corte transversal, las varianzas se ven afectadas por los valores de las variables, cumpliéndose el fenómeno llamado heteroscedasticidad.

Esta metodología también ha sido utilizada en trabajos como en Estrada (2001) y Lima et al. (2010). La heteroscedasticidad se presenta frecuentemente en las estimaciones con datos tipo panel, como señala Estrada (2001), y repercute en los estimadores que, aunque son insesgados y consistentes, no son eficientes; esto origina sesgos en el cálculo de las varianzas muestrales y en la aplicación incorrecta de los contrastes de significancia estadística, por lo que en esta investigación MCG sería más conveniente que MCO. Por último, para estimar los coeficientes del modelo de regresión se empleó el programa $e$-views.

\section{Resultados}

\section{Estadísticas}

Hemos mencionado que las empresas seleccionadas han tenido el distintivo al menos un año y además cotizan en la BMV; en total suman 19 y se encuentran clasificadas en seis sectores. En la figura 2 presentamos la composición de la muestra por sectores. El sector con mayor participación en la muestra es el sector de transformación, con un $47 \%$ del total de la muestra, y con menos representación tenemos los sectores de transportación y de extracción con sólo un 5\%.

\section{Figura 2}

\section{Ponderación de los sectores bajo estudio}

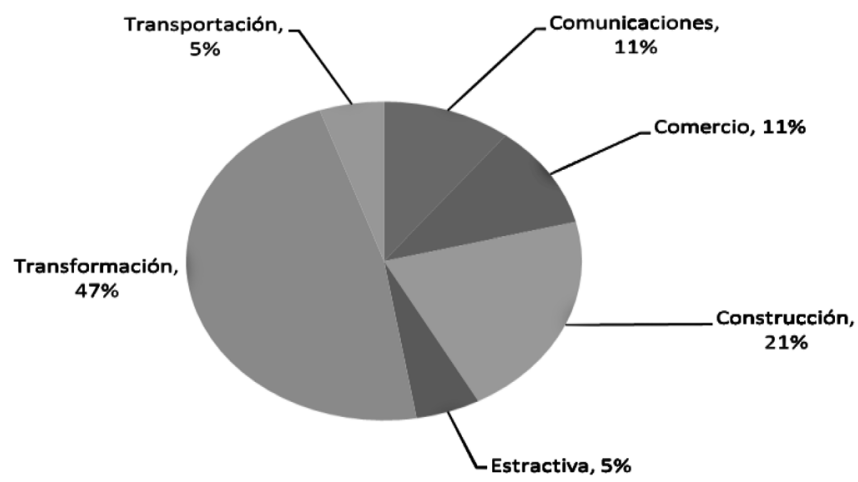




\section{Cuadro 2}

\section{Estadísticas por sector}

\begin{tabular}{|c|c|c|c|c|}
\hline Variables & Mean & Maximum & Minimum & Std. Dev. \\
\hline Comunicaciones & & $n=2$ & & \\
\hline ROA & $8 \%$ & $21 \%$ & $-3 \%$ & $7 \%$ \\
\hline ROE & $27 \%$ & $84 \%$ & $-9 \%$ & $26 \%$ \\
\hline Crecimiento en Utilidade & $-24 \%$ & $135 \%$ & $-243 \%$ & $91 \%$ \\
\hline$P / V L$ & 2.3 & 7.0 & 0.6 & 1.9 \\
\hline P/UPA & 14.8 & 76.2 & 0.0 & 21.3 \\
\hline Rendimiento en precios & $-7 \%$ & $60 \%$ & $-264 \%$ & $84 \%$ \\
\hline RSC & 0.83 & 1.00 & 0.00 & 0.39 \\
\hline Tamaño (log Activos) & 0.07 & 0.75 & -0.37 & 0.26 \\
\hline Riesgo (P/A) & 0.64 & 0.79 & 0.55 & 0.09 \\
\hline Transformación & & $n=9$ & & \\
\hline ROA & $4 \%$ & $10 \%$ & $-8 \%$ & $5 \%$ \\
\hline ROE & $10 \%$ & $23 \%$ & $-12 \%$ & $9 \%$ \\
\hline Crecimiento en Utilidade & $3 \%$ & $365 \%$ & $-177 \%$ & $94 \%$ \\
\hline$P / V L$ & 2.36 & 9.19 & 0.15 & 2.47 \\
\hline P/UPA & 15.62 & 117.13 & 0.00 & 24.19 \\
\hline Rendimiento en precios, & $-3 \%$ & $67 \%$ & $-215 \%$ & $68 \%$ \\
\hline RSC & 0.38 & 1.00 & 0.00 & 0.49 \\
\hline Tamaño (log Activos) & 0.19 & 0.61 & -0.23 & 0.18 \\
\hline Riesgo (P/A) & 0.58 & 0.73 & 0.44 & 0.07 \\
\hline Comercio & & $n=2$ & & \\
\hline ROA & $8 \%$ & $16 \%$ & $-1 \%$ & $6 \%$ \\
\hline ROE & $12 \%$ & $26 \%$ & $-1 \%$ & $9 \%$ \\
\hline Crecimiento en Ut & $60 \%$ & $1387 \%$ & $-253 \%$ & $362 \%$ \\
\hline$P / V L$ & 1.31 & 3.20 & 0.48 & 0.95 \\
\hline P/UPA & 21.03 & 157.17 & 0.00 & 37.12 \\
\hline Rendimiento en Precios & $4 \%$ & $52 \%$ & $-80 \%$ & $38 \%$ \\
\hline RSC & 0.56 & 1.00 & 0.00 & 0.51 \\
\hline Tamaño (log Activos) & 0.04 & 0.21 & -0.30 & 0.11 \\
\hline Riesgo (P/A) & 0.37 & 0.46 & 0.17 & 0.08 \\
\hline Transporte & & $n=1$ & & \\
\hline ROA & $4 \%$ & $6 \%$ & $2 \%$ & $1 \%$ \\
\hline ROE & $4 \%$ & $7 \%$ & $2 \%$ & $2 \%$ \\
\hline Crecimiento en Ut & $21 \%$ & $109 \%$ & $-24 \%$ & $54 \%$ \\
\hline$P / V L$ & 0.84 & 1.39 & 0.33 & 0.33 \\
\hline P/UPA & 21.37 & 36.59 & 12.33 & 7.56 \\
\hline Rendimiento en Precios & $11 \%$ & $42 \%$ & $-30 \%$ & $27 \%$ \\
\hline RSC & 0.13 & 1.00 & 0.00 & 0.35 \\
\hline Tamaño (log Activos) & 0.02 & 0.08 & -0.04 & 0.04 \\
\hline Riesgo (P/A) & 0.09 & 0.17 & 0.05 & 0.05 \\
\hline Extractiva & & $n=1$ & & \\
\hline ROA & $8 \%$ & $14 \%$ & $-1 \%$ & $6 \%$ \\
\hline ROE & $15 \%$ & $30 \%$ & $-1 \%$ & $12 \%$ \\
\hline Crecimiento en Ut & $-311 \%$ & $118 \%$ & $-1854 \%$ & $703 \%$ \\
\hline$P / V L$ & 2.94 & 6.09 & 0.90 & 1.56 \\
\hline P/UPA & 20.88 & 73.80 & 0.00 & 24.54 \\
\hline Rendimiento en Precios & $29 \%$ & $62 \%$ & $-36 \%$ & $34 \%$ \\
\hline RSC & 1.00 & 1.00 & 1.00 & 0.00 \\
\hline Tamaño (log Activos) & 0.12 & 0.47 & -0.01 & 0.17 \\
\hline Riesgo (P/A) & 0.47 & 0.56 & 0.36 & 0.06 \\
\hline Construcción & & $n=4$ & & \\
\hline ROA & $4 \%$ & $8 \%$ & $14 \%$ & $-1 \%$ \\
\hline ROE & $10 \%$ & $18 \%$ & $-3 \%$ & $-1 \%$ \\
\hline Crecimiento en Ut & $6 \%$ & $558 \%$ & $-78 \%$ & $278 \%$ \\
\hline$P / V L$ & 2.16 & 4.82 & 0.54 & 1.79 \\
\hline P/UPA & 13.98 & 42.74 & 1.78 & 15.05 \\
\hline Rendimiento en Precios & $29 \%$ & $101 \%$ & $-56 \%$ & $56 \%$ \\
\hline RSC & 0.31 & 1.00 & 0.00 & 0.43 \\
\hline Tamaño (log Activos) & 7.57 & 7.91 & 7.29 & 3.91 \\
\hline Riesgo (P/A) & 0.58 & 0.66 & 0.49 & 0.06 \\
\hline
\end{tabular}


En el cuadro 2, se presentan las estadísticas de algunas variables del modelo. Cabe destacar que la antigüedad promedio del distintivo de RSE de la muestra es de tres años con un ROE de $11 \%$ y una desviación del ROE muy significativa de 9.3\%; esto es, existe una gran desviación entre las empresas bajo estudio debido a que son de diferentes tamaños y sectores. En relación con el P/VL, este indicador nos reporta en promedio 1.8, lo que indica que la muestra se encuentra sobrevalorada en relación con el valor en libros.

Los sectores de comunicación, comercio y extracción tienen un ROA de $8 \%$, mientras que el resto alcanzan apenas el 4\%. Por otra parte, el ROE más alto es el del sector de comunicación. En lo que respecta al rendimiento del precio de la acción, el sector de la industria extractiva tiene un rendimiento de $29 \%$ y esto se refleja con un múltiplo de P/VL de 3, lo que señala que el mercado tiene un pronóstico positivo en el valor de la empresa. Cabe destacar que la desviación en el rendimiento de las acciones se refleja en los sectores de comunicaciones y transformación con $84 \%$ y $68 \%$, respectivamente, y cuyos rendimientos promedios son negativos.

Para este modelo econométrico, consideramos que no podríamos ser restrictivos en cuanto a la elección del parámetro de desempeño financiero; así, consideramos tres tipos de variables para el desempeño financiero: contables, mixtas y de mercado. A partir de esto, mostramos resultados obtenidos en los cuadros 3 a 5 .

\section{Cuadro 3}

Modelo de los efectos de la RSE en el desempeño financiero basado en medidas contables: Rendimiento sobre Activos (ROA), Rendimiento sobre Capital (ROE), las Utilidades por Acción (UPA) y el crecimiento de las utilidades

\begin{tabular}{|c|c|c|c|c|c|}
\hline & \multicolumn{5}{|c|}{ Variables Independiente } \\
\hline Variable Dependiente & RSE & Tamaño & Riesgo & Durbin & $\mathrm{R}$ cuadrada ajustada \\
\hline ROE & RSE & Activo & $\mathrm{P} / \mathrm{A}$ & & \\
\hline Beta & 0.030269 & 0.007444 & -0.03959 & 0.85954 & 0.18037 \\
\hline pvalue & 0.000000 & 0.000000 & $0.1452^{*}$ & & \\
\hline ROA & RSE & Activo & $\mathrm{P} / \mathrm{A}$ & & \\
\hline Beta & 0.02327 & 0.005947 & -0.098082 & 0.65187 & 0.3105 \\
\hline pvalue & 0.0000 & 0.0000 & 0.0000 & & \\
\hline UPA & RSE & Activo & $\mathbf{P} / \mathbf{A}$ & & \\
\hline Beta & 0.8427 & 0.1295 & -2.3145 & 0.8272 & 0.2763 \\
\hline p value & 0.000000 & 0.000000 & 0.000000 & & \\
\hline Crecimiento en Utilidades & RSE & Activo & $\mathrm{P} / \mathrm{A}$ & & \\
\hline Beta & 0.038547 & -0.0066 & -0.0578 & 2.2337 & 0.00011 \\
\hline pvalue & $0.3345^{*}$ & 0.0208 & $0.6119^{*}$ & & \\
\hline
\end{tabular}


Los resultados del cuadro 3 muestran que de las cuatro estimaciones casi todas las betas de la variable de RSE son estadísticamente significativas, excepto en crecimiento de utilidades. Además, la variable de desempeño financiero que mejor explica la relación con el distintivo de RSE es UPA porque impacta en 0.8427 , contrario a los resultados de Lima et al. (2010). Siguiendo con este mismo caso, el tamaño de la empresa medido por el logaritmo natural de los activos es estadísticamente significativo y positivo, mientras que el riesgo medido por la razón de P/A es negativo y tiene un gran impacto en la UPA, igual que en Lima et al. (2010). Los resultados de este artículo en este punto son similares a los de Peters y Mullen (2009), pues estos autores, para el caso americano, encuentran relaciones positivas entre RSE y ROA.

\section{Cuadro 4}

Modelo de los efectos de la RSE en el desempeño financiero basado en medidas en medidas mixtas o múltiplos: precio entre valor en libros (P/VL) y el precio entre utilidades por acción (P/UPA).

\begin{tabular}{|c|c|c|c|c|c|}
\cline { 2 - 6 } \multicolumn{1}{c|}{} & \multicolumn{5}{c|}{ Variables Independiente } \\
\hline Variable Dependiente & RSE & Tamaño & Riesgo & Durbin & R cuadrada ajustada \\
\hline P/NL & RSE & Activo & P/A & & \\
Beta & $\mathbf{0 . 5 8 8 2}$ & $\mathbf{0 . 0 4 2 6}$ & $\mathbf{1 . 5 8 0 6}$ & $\mathbf{0 . 8 5 7 3}$ & $\mathbf{0 . 3 0 2 7}$ \\
p value & $\mathbf{0 . 0 0 0 0}$ & $\mathbf{0 . 0 0 0 0}$ & $\mathbf{0 . 0 0 0 1}$ & & \\
\hline P/UPA & RSE & Activo & P/A & & \\
Beta & 0.1328 & 1.227 & -0.18744 & 1.455 & 0.1767 \\
p value & $0.91^{*}$ & 0.0000 & 0.0000 & & \\
\hline
\end{tabular}

En estas estimaciones, el mejor modelo es cuando utilizamos la variable de desempeño financiero $\mathrm{P} / \mathrm{VL}$, ya que la RSE, el tamaño y el riesgo son estadísticamente significativos y diferentes de cero. En cambio, no se encuentra impacto de RSE en el desempeño financiero si usamos como indicador la razón de P/UPA. Los resultados son inversos a los reportados por Lima et al. (2010), pero similares a los de Peters y Mullen (2009).

\section{Cuadro 5}

Modelo de los efectos de la RSE en el desempeño financiero basado en medidas de mercado: rendimiento sobre precios.

\begin{tabular}{|c|c|c|c|c|c|}
\cline { 2 - 6 } \multicolumn{1}{c|}{} & \multicolumn{5}{c|}{ Variables Independiente } \\
\hline Variable Dependiente & RSE & Tamaño & Riesgo & Durbin & R cuadrada ajustada \\
\hline Rendimiento de mercado & RSE & Activo & P/A & & \\
Beta & 0.06192 & -0.01298 & 0.0705 & 2.217 & 0.01547 \\
p value & $0.1446^{*}$ & 0.0001 & $0.5718^{*}$ & & \\
\hline
\end{tabular}


Por último, encontramos que la RSE y el rendimiento de mercado no tienen dependencia y que el tamaño del activo está inversamente relacionado con el desempeño de la empresa; en cuanto al riesgo tenemos un coeficiente positivo y no significativo. Los resultados son parecidos a los reportados por Lima et al. (2010), a excepción del riesgo.

Para validar el supuesto de normalidad de los resultados (cuadros 3, 4 y 5) se probó si los residuos generados de cada modelo por empresa seguían una distribución normal. Para ello, se aplicó el test propuesto por Jarque y Bera (1980) que se basa en el estudio de la forma que tiene la distribución de frecuencias, examinando sus discrepancias respecto a la curva campaniforme característica del modelo normal. Estas discrepancias respecto a la normalidad deben ser evaluadas según dos características de forma: la simetría $\left(\gamma_{1}\right)$ y la curtosis $\left(\gamma_{2}\right)$. El estadístico de Jarque y Bera resume las características de forma de la muestra y adoptará valores bajos si la distribución observada es aproximadamente simétrica y mesocúrtica. Los resultados del análisis concluyeron que los residuos seguían una distribución normal.

En definitiva, los resultados muestran que existe una relación positiva entre el desempeño financiero y la RSE. De todos los modelos propuestos, el que mejor se ajustó considerando la R cuadrada ajustada, es cuando incluimos UPA y P/VL. En Lima et al. (2010), se utiliza el precio entre valor en libros y el ROE como variable de desempeño financiero, mientras que el riesgo como pasivo entre activo, el tamaño de la empresa como el logaritmo de los activos y la RSE (índice de mercado tipo KDL) se usan como variables independientes. Los resultados muestran que la RSE está destruyendo valor en Brasil, ya que se encontró una correlación negativa entre la responsabilidad social y el valor de firma medido por precio entre valor en libros y ROA.

\section{Conclusiones}

La literatura ha puesto de manifiesto que la mejora del desempeño financiero, de forma directa o indirecta a través de la mejora de la imagen de la empresa u otros beneficios intangibles, es una de las principales razones que explican la adopción de prácticas de RSE (Annandale y Taplin, 2003; Bull, 2003; Kiernan, 2001; Prakash, 2000; Sharma, 2000; Tullberg, 2005). Sin embargo, hasta ahora no se había verificado en México si las empresas comprometidas con la RSE y certificadas obtenían mejoras financieras. 
Los resultados obtenidos muestran los efectos positivos que en la empresa cotizada tiene el distintivo de RSE, puesto que resulta significativo su impacto, especialmente en las variables de desempeño financiero de valor de mercado. Estos resultados deben animar a las empresas a continuar e incrementar su actividad de RSE (Kang et al., 2010).

Es interesante el hecho de que un $15 \%$ de las empresas que cotizan en la BMV y que han alcanzado el distintivo son de sectores manufactureros o extractivos, con un alto impacto social positivo, pues proporcionan muchos empleos directos e indirectos generando riqueza en su área de influencia, pero también negativo al generar contaminación medioambiental. Esto puede sugerir que estas empresas, que tienen una alta visibilidad, quieren mandar un mensaje explícito al mercado sobre su comportamiento social y compromiso con la sociedad donde están localizadas.

El hecho que sea precisamente en las variables de desempeño financiero de valor de mercado sugiere que cuando se obtiene el distintivo éste actúa como un anuncio al mercado de que la empresa está contribuyendo a mejorar su entorno, satisfacer las expectativas de sus stakeholders e incrementar la riqueza en su comunidad. Por lo tanto, los inversionistas consideran que esta empresa se esfuerza por tener un futuro sostenible y premian esta actividad con rendimientos anormales (Nicolau, 2008). Estos resultados también sugieren que las empresas mexicanas distinguidas con el distintivo están acertando en proporcionar a sus stakeholders aquellas acciones que aportan valor. Las empresas mexicanas deben desarrollar su propia identidad en la adopción de RSE porque la imitación de prácticas de RSE que se están desarrollando en otros países como Estados Unidos o Europa pueden no satisfacer a los stakeholders de las empresas mexicanas, puesto que la identidad e intereses de éstos varía de acuerdo con la nacionalidad (Matten y Moon, 2008).

Los autores anteriores también han afirmado que el desarrollo de índices de inversión socialmente responsable puede motivar a las empresas que tienen necesidad de acceder a capital externo a desarrollar acciones de RSE; pero hay que evaluar si realmente estas acciones están penetrando en la cultura de la empresa a largo plazo o sólo es un maquillaje para acceder a capital externo adicional para hacer crecer el negocio. Los resultados de nuestro estudio muestran que las empresas con el distintivo tienen una más alta capitalización, con lo que probablemente estén en buena disposición para conseguir financiación externa que les permita desarrollar sus planes de desarrollo futuro. 
Las conclusiones anteriores nos llevar a proponer algunas recomendaciones para la práctica empresarial. En primer lugar, puesto que el desarrollo de la RSE por parte de la empresa está relacionado con un mayor desempeño financiero, las empresas mexicanas deberían esforzarse por introducirla dentro de su estrategia y cultura empresarial. En segundo lugar, las empresas mexicanas deben desarrollar su propia identidad en RSE, basada en su entorno y sus stakeholders, sin imitar prácticas desarrolladas en otros entornos distintos, lo que implica un análisis detallado de sus stakeholders y sus expectativas, y alinearlas con la estrategia de la empresa. Finalmente, se recomienda tener una política de RSE clara, visible, sostenida y certificada por un tercero, pues la misma puede optimizar los planes de desarrollo de la empresa.

Además, aunque es una práctica relativamente joven en Latinoamérica, todavía resulta más relevante, si cabe, el compromiso de estas empresas con la RSE porque los gobiernos de los países latinoamericanos no han resultado ser históricamente "sociales", por eso - aun ahora- son necesarias políticas sociales adaptadas a cada país, que permitan que las empresas puedan apoyar y contribuir con su granito de arena al desarrollo comunitario de una forma "personalizada" a cada entorno. Aunque las empresas con dicho distintivo lo han mantenido durante años, no se ve un crecimiento significativo de las empresas que trabajan por alcanzarlo. Esto puede ser debido a la falta de apoyo "explícito" del gobierno, que debe generar el suficiente respaldo o ambiente institucional para que las empresas tomen la decisión de desarrollar de forma deliberada, voluntaria e "inducida" sus políticas, normas y acciones de RSE (Porter y Kramer, 2006).

Por lo tanto, se recomienda a los legisladores trabajar en dos vías de actuación. La primera de ellas sería el desarrollo de campañas de sensibilización y foros de discusión sobre la RSE, para todo tipo de empresas, especialmente para las medianas y pequeñas, ya que todas las empresas pueden potenciar la responsabilidad empresarial independientemente de su tamaño. La segunda vía de actuación sería la adopción de políticas y recomendaciones que faciliten que las empresas puedan adoptar acciones de responsabilidad social dentro de su ámbito de actuación.

Como conclusión, se puede afirmar a la vista de los resultados obtenidos, que las empresas mexicanas, al menos las cotizadas, deben marcar como objetivo estratégico el incorporar la RSE a la estrategia de la empresa desde la realidad y necesidad de México. Sin duda, esto les traerá grandes beneficios económicos, de desarrollo futuro y de imagen de empresa. 
Sin embargo, este artículo tiene varias limitaciones que los autores pretenden subsanar en próximos estudios. En primer lugar, es necesario ampliar la muestra a las empresas que no cotizan en bolsa, pero que han obtenido el distintivo; en segundo lugar, determinar el tipo de actividades de RSE que están realizando las empresas mexicanas y su contribución real a su entorno; en tercer lugar, identificar las diferencias existentes en México en las prácticas de RSE entre las empresas de matriz mexicana y de otras nacionalidades. Finalmente, se pretende categorizar las prácticas de RSE específicas que realizan las empresas mexicanas y compararlas con las del resto del mundo.

\section{Referencias}

Accountability (2003). AA1000 Norma sobre aseguramiento. Londres: Accountability.

Alonso-Almeida, M.M. (2007). El gobierno corporativo electrónico. Análisis desde el enfoque de confianza. Madrid: Visión Libros.

(2009). Utilización de las tecnologías de la información para generar confianza de los accionistas e inversores en las empresas. Argos 26 (51): 6-26.

Annandale, D. y R. Taplin (2003). The determinants of mining company response to environmental approvals regulation: A report of australian research. Journal of Environmental Planning and Management 46 (6): 887-909.

Aras, G., A. Aybar y O. Kutlu (2010). Managing corporate performance: investigating the relationship between corporate social responsibility and financial performance in emerging markets. International Journal of Productivity and Performance Management 59 (3): 229-254.

Aupperle, K. E., A. B. Carroll, y J. D. Hatfield (1985). An empirical examination of the relationship between Corporate Social Responsibility and profitability. Academy Of Management Journal 28 (2): 446-463.

Barroso, F. (2008). La Responsabilidad Social Empresarial. Un estudio en 40 empresas de la ciudad de Mérida Yucatán. Contaduría y Administración (226): 73-91. 
Bartels, K. K., E. Harrick, K. Martell y D. Strickland (1998). The relationship between ethical climate and ethical problems within human resource management. Journal of Business Ethics 17 (7): 799-804.

Bragdon, J. y J. A. Marlin (1972). Is pollution profitable? Environmental virtue and reward: must stiffer pollution controls hurt profits? Risk Management (19): 9-18.

Brammer, S., C. Brooks y S. Pavelin (2006). Corporate social performance and stock returns: UK evidence from disaggregate measures. Financial Management, Autumn: 97-116.

Bueno-Campos, E. (Ed.) (2004). El gobierno de la empresa. En busca de la transparencia y la confianza. Madrid: Editorial Pirámide.

Bull, B. (2003). Corporate Social Responsibility: The norwegian experience. Paper prepared for the initiative on ethics and development. The Inter-american development bank. www.iadb.org.

Clarkson, M. B. E. (1995). A stakeholder framework for analyzing and evaluating corporate social performance. Academy of Management Review 20 (1): 92-117.

Dierkes M. y A. B. Antal (1985). The usefulness and use of social reporting information. Accounting, organizations and society 10 (1): 29-34.

Derwall, J., N. Guenster, R. Bauer y K. Koedijk (2005). The eco-efficiency premium puzzle. Financial Analysts Journal (61): 51-63.

Estrada, J. (2001). "The cost of equity in emerging markets: A downside risk approach (II)”. Emerging Markets Quarterly 5 (1): 63-72.

Freeman, R.E. (1994). The politics of stakeholder theory: some future directions. Business Ethics Quarterly 4 (4): 409-422.

Friedman, M. (1970). The social responsability of business is toincrease its profits. New York Times Magazine, 13 de septiembre: 122-126. 
Press.

(1962). Capitalism and freedom. Chicago: The University of Chicago

Graves, S. B. y S. A. Waddock (1994). Institutional owners and corporate social. Academy of Management Journal (37): 1034-1046.

Greenwood, M. (2007). Stakeholder engagement: beyond the myth of corporate responsibility. Journal of Business Ethics 74 (4): 315-327.

GRI (2009). Guia para la elaboración de memorias de sostenibilidad. Disponible en http://www.globalreporting.org/

Griffin, J. y J. Mahon (1997). The corporate social and corporate financial debate: twenty-five years of incomparable research. Business and Society 36 (1): $5-31$.

Haigh, M. y M. T. Jones (2006). The drivers of corporate social responsibility: a critical review. Business Review 5 (2): 245-251.

Igalens, J. y J.P. Gond (2005). Measuring corporate social performance in france: a critical and empirical analysis of ARESE Data. Journal of Business Ethics 56 (2): 131-148.

ISO (2006). Norma ISO 26000: Guía sobre responsabilidad social. Boletin IRAM, junio: 2-3.

Jarque, C. M. y A. K. Bera (1980). Eficient tests for normality, homoscedasticity and serial independence of regression residuals. Econometrics Letters 6 (3): 255-259.

Kiernan, M. (2001). Eco-value, sustainability and shareholder value: driving environmental performance to the bottom line. Environmental Quality Management, Summer: 1-12.

Kang, K. H, S. Lee y C. Huh (2010). "Impacts of positive and negative corporate social responsibility activities on company performance in the hospitality industry”. International Journal of Hospitality Management (29): 72-82. 
Kramer, R. M. y T. R. Tyler (1996). Trust in organizations. Frontiers of theory and research. Londres: Sage Publications.

Lane, C. y R. Bachmann (2000). Trust within and between organizations: conceptual and issues and empirical applications. Oxford: Oxford University Press.

Lee, D D. y R. W. Faff (2009). Corporate sustainability performance and idiosyncratic risk: a global perspective. The Financial Review 44 (2): 213-237.

Lima, Vicente F., F. de Souza y F. C. Vasconcellos (2010). Corporate social responsibility, firm value and financial performance in Brazil, Social Responsibility Journal (forthcoming). Disponible en SSRN: http://ssrn.com/abstract $=1587023$

Maignan, I. y O. C. Ferrell (2000). Measuring corporate citizenship in two countries: the case of the United States and France. Journal of Business Ethics 23 (3): 283-297.

y G. T. M. Hult (1999). Corporate citizenship: cultural antecedents and business benefits. Journal of the Academy of Marketing Science 27: 455-459.

Margolis, J. D. y J. P. Walsh (2001). Social enterprise series No. 19 --Misery loves companies: whither social initiatives by business?; Harvard Business School Working Paper Series: 01-058.

H. Elfenbein y J. Walsh (2007). Does it pay to be good? A metaanalysis and redirection of research on the relationship between corporate social and financial performance. Harvard Business School: Working Paper.

Matten D. y J. Moon (2008). "Implicit" and "Explicit" CSR:a conceptual framework for a comparative understanding of corporate social responsibility. Academy of Management Review 33 (2): 404-424.

Méndez Picazo, M.T. (2005). Ética y responsabilidad social corporativa. Cuadernos ICE, junio: 141-150. 
Muller A. y A. Kolk (2009). CSR in emerging markets evidence from Mexico. Journal of Business Ethics (85) Suplement: 325-337.

Nicolau, J.L. (2008). Corporate Social Responsibility: worth-creating activities. Annals of Tourism Research 35 (4): 990-1006.

Orlitzky, M. (2007). Doing well by doing good: objective findings, subjective assumptions or selective amplification? Paper presented at the Academy of Management Conference, Philadelphia.

, F. Schmidt y S. Rynes (2003). Corporate social and financial performance: a meta-analysis. Organization Studies 24 (3): 403-41.

Peloza, J. (2009). The challenge of measuring financial impacts from investments in corporate social performance. Journal of Management 35 (6): 1518-1541.

Peters, R. y M. Mullen (2009). Some evidence of cumulative effects of corporate social responsibility on financial. Journal of Global Business 3 (1): 1-14.

Petersen, H. y H. Vredenburg (2009). Morals or economics? Institutional investor preferences for corporate social responsibility. Journal of Business Ethics (90): $1-14$.

Porter, M. E. y M. R. Kramer (2006). Strategy and society: the link between competitive advantage and corporate social responsibility. Harvard Business Review 84 (12): 78-92.

Porto, N. y J. Castromán (2006). Responsabilidad social: un análisis de la situación actual de México y España. Contaduría y Administración (220): 67-88.

Post, J.E., L.E. Preston y S. Sachs (2002). Managing the extended enterprise: the new stakeholder view. California Management Review 45 (1): 6-28.

Prakash, A. (2000). Greening the firm: the politics of corporate environmentalism. Cambridge: Cambridge University Press. 
Rappaport, A. (1998). La creación de valor para el accionista: una guía para inversores y directivos. Bilbao: Deusto.

Schnietz, K. E., y M. J. Epstein (2005). Exploring the financial value of a reputation for corporate social responsibility during a crisis. Corporate Reputation Review (7): 327-345.

Sharma, S., (2000). Managerial interpretations and organizational context as predictors of corporate choice of environmental strategy. Academy of Management Journal 43 (4): 681-697.

Tullberg, J. (2005). Reflections upon the responsive approach to corporate social responsibility. Business Ethics: A European Review 14 (3): 261-276.

Ullman, A. A. (1985). Data in search of a theory: a critical examination of the relationships among social performance, social disclosure and economic performance of US firms. The Academy of Management Review 10 (3): 540-557.

Waddock, S. (2004). Parallel universes: companies, academics, and the progress of corporate citizenship. Business and Society Review (109): 15-42

y S. Graves (1997). The corporate social perdorm link. Strategic Management Journal (18): 303-317.

Wiggins, R.R. y T. W. Ruefli (2002). Sustained competitive advantage: temporal dynamics and the incidence and persistence of superior economic financial performance. Organization Science 13 (1): 82-104.

Winstanley, D., J. Woodhall y E. Heery (1996). The agenda for ethics in human resource management. Business ethics: a european review 5 (4): 187-194.

Wood, D. J. (1991). Corporate social performance revisited. Academy of Management Review 16 (4): 691-718. (A) 
